\title{
Disrupted Brain Entropy And Functional Connectivity Patterns Of Thalamic Subregions In Major Depressive Disorder
}

This article was published in the following Dove Press journal: Neuropsychiatric Disease and Treatment

\author{
Shao-Wei Xue ${ }^{1,2}$ \\ Donglin Wang ${ }^{1,2}$ \\ Zhonglin $\operatorname{Tan}^{3}$ \\ Yan Wang ${ }^{1,2}$ \\ Zhenzhen Lian ${ }^{1,2}$ \\ Yunkai Sun ${ }^{1,2}$ \\ Xiaojiao $\mathrm{Hu}^{1,2}$ \\ Xiaole Wang ${ }^{1,2}$ \\ Xin Zhou ${ }^{1,2}$
}

'Center for Cognition and Brain Disorders, Institutes of Psychological Sciences and the Affiliated Hospital, Hangzhou Normal University, Hangzhou 3 I I 2 I, People's Republic of China; ${ }^{2}$ Zhejiang Key Laboratory for Research in Assessment of Cognitive Impairments, Hangzhou 31 II2I, People's Republic of China; ${ }^{3}$ Department of Psychiatry, Hangzhou Seventh People's Hospital, Hangzhou 310013, People's Republic of China
Correspondence: Donglin Wang Center for Cognition and Brain Disorders, Institutes of Psychological Sciences and the Affiliated Hospital, Hangzhou Normal University, 2318

Yuhangtang road, Hangzhou, Zhejiang

3 III2I, People's Republic of China

Tel +86 57I 28867717

Fax +86 57I 28867717

Email wangdl@hznu.edu.cn
Purpose: Entropy analysis of resting-state functional magnetic resonance imaging (R-fMRI) has recently been adopted to characterize brain temporal dynamics in some neuropsychological or psychiatric diseases. Thalamus-related dysfunction might be a potential trait marker of major depressive disorder (MDD), but the abnormal changes in the thalamus based on R-fMRI are still unclear from the perspective of brain temporal dynamics. The aim of this study was to identify local entropy changes and subregional connectivity patterns of the thalamus in MDD patients.

Patients and methods: We measured the sample entropy of the R-fMRI data from 46 MDD patients and 32 matched healthy controls. We employed the Louvain method for the module detection algorithm to automatically identify a functional parcellation of the thalamus and then examined the whole-brain subregional connectivity patterns.

Results: The results indicated that the MDD patients had decreased entropy in the bilateral thalami compared with healthy controls. Increased functional connectivity between the thalamic subregions and the medial part of the superior frontal gyrus (mSFG) was found in MDD patients.

Conclusion: This study showed new evidence about sample entropy changes in MDD patients. The functional connectivity alterations that were widely distributed across almost all the thalamic subregions with the mSFG in MDD suggest a general involvement independent of the location and function of the subregions.

Keywords: major depressive disorder, entropy, thalamus, functional connectivity, superior frontal gyrus

\section{Introduction}

Major depressive disorder (MDD) is a commonly encountered psychological or psychiatric disorder characterized by pervasive and persistent low mood, accompanied by physical symptoms, cognitive dysfunction, and impaired social function. ${ }^{1}$ This disorder, with its high levels of morbidity and mortality, contributes to increased suicide rates and various unfavourable medical outcomes. ${ }^{2}$ The underlying pathology of MDD is under continuous investigation, although there is now increasing evidence for biological, psychological, and social factors contributing to MDD. ${ }^{3}$ Magnetic resonance imaging (MRI) techniques have contributed greatly to the identification of neurobiological correlates of MDD in recent years and provided converging evidence in support of neuropathology in this disorder. ${ }^{4-6}$ Many studies have shown that thalamic abnormalities might be potential neuroimaging-based markers of MDD. ${ }^{7,8}$ 
The characteristic clinical symptoms and associated cognitive deficits of MDD may arise, in part, through the corresponding dysfunction of thalamus-related brain circuits. ${ }^{9}$

The thalamus is a subcortical structure located near the brain centre, with nerve fibres projecting into multiple directions. This region is a relay for - and an integration hub of - neural pathways, including the cortico-basal ganglia-thalamo-cortical loop or thalamo-cortical system. ${ }^{10,11}$ Anatomically, the thalamus is a complex structure organized in many nuclei subserving various functions, and these have highly selective connectivity patterns with some specific areas. ${ }^{12,13}$ For example, the dorsal thalamic nuclei integrate and relay sensory information to the cerebral cortex. The anterior thalamic nuclei have reciprocal connections with the cingulate gyrus and hypothalamus. However, aberrations in these relay connections of the thalamus have also been reported in MDD. ${ }^{14}$ Considering the thalamic heterogeneity and its association with MDD neuropathology, ${ }^{12,15}$ it is inadequate to examine functional deficits from the perspective of a single region of interest (ROI) in the thalamus. Previous studies divided the thalamus into several parts to examine the functional specialization of different thalamic subregions. ${ }^{14,16}$ Kong et $\mathrm{al}^{16}$ partitioned the whole cortex into six large areas, namely, the prefrontal, motor, somatosensory, parietal, temporal, and occipital cortex, and used them as seeds to examine functional connectivity patterns with each thalamic voxel. Based on specific connectivity patterns of each cortical subdivision, they identified six thalamic parcellations and then examined their whole-brain connectivity patterns. They found MDD-related abnormalities in the connectivity patterns of the prefrontal, motor, somatosensory, and parietal thalamic subdivisions, with both hyperconnectivity and hypoconnectivity. One issue of this study was that the six areas of the whole cortex were not well defined and might affect functional parcellation within the thalamus.

Entropy is usually described as the degree of uncertainty or disorder in a system that is a property of the system's state. In recent years, this method provides a means to quantify resting-state functional MRI (R-fMRI) data presenting ongoing fluctuating activity in the brain, ${ }^{17}$ and it further facilitates research on the potential functional architecture of the brain. ${ }^{18}$ This temporal brain entropy with high levels of entropy corresponds to low levels of predictability and high levels of randomness. ${ }^{19}$ Based on R-fMRI data acquired from 1049 subjects and statistical threshold ( $\mathrm{p}<0.01$, corrected for multiple comparison), Wang et al have shown reliably distributed brain entropy patterns in the normal brain, such as the hierarchical structure. ${ }^{20}$ The brain entropy dynamics were altered in neuropsychological or psychiatric conditions, including schizophrenia, ${ }^{21}$ Alzheimer's disease, ${ }^{22}$ relapsing-remitting multiple sclerosis, ${ }^{23}$ and attention-deficit/hyperactivity disorder. ${ }^{24}$ However, it remains unknown whether the entropy patterns of thalamic subregions are differentially disrupted in MDD.

In the present study, we were to locate the entropy alterations across the whole brain in MDD patients. Meanwhile, automated functional parcellation of the thalamus was performed using a module detection algorithm based on all pairwise correlation coefficients between the voxel time series and the resting-state functional connectivity (RSFC) of each thalamic parcellation was also traced. Considering that the thalamus was significantly involved in $\mathrm{MDD}^{25}$ which is often manifested in abnormal local regional activity ${ }^{26}$ and dysregulation in the thalamo-cortical system, ${ }^{14}$ we hypothesized that MDD patients showed disrupted brain entropy in the thalamus and abnormal thalamic RSFC with cerebral regions.

\section{Materials and Methods \\ Participants}

Forty-six patients with MDD (age: 28.32 \pm 8.55 yrs, 13 males/33 females) and 32 sex- and age-matched healthy controls (HC, age: $27.19 \pm 10.29$ yrs, 10 males/22 females) were recruited from the Department of Psychiatry of Hangzhou Seventh People's Hospital and the Department of Psychiatry at the Affiliated Hospital of Hangzhou Normal University. All subjects were right-handed according to the Edinburgh Handedness Inventory. ${ }^{27}$ MDD subjects were diagnosed based on the Diagnostic and Statistical Manual of Mental Disorders, Fourth Edition (DSM-IV) criteria using the Mini-Neuropsychiatric International Interview (MINI). The demographic data and clinical characteristics of the participants are listed in Table 1.

All patients were interviewed by certified psychiatrists, and the severity of depressive symptoms was evaluated using the Hamilton Depression Rating Scale (HDRS; Hamilton, 1967). Participants were administered the Life Events Scale (LES) to assess the degree of enjoyment and satisfaction and the Ways of Coping Checklist (WCCL) to assess thoughts and behaviours related to coping strategies during stressful events. The exclusion criteria for the study included the following: 1) current pregnancy or breastfeeding; 2) severe 
Table I Demographic And Clinical Data

\begin{tabular}{|l|l|l|l|}
\hline & $\begin{array}{l}\text { MDD } \\
\text { (N= 46) } \\
\text { Mean (SD) }\end{array}$ & $\begin{array}{l}\text { HC } \\
\mathbf{( N = 3 2 )} \\
\text { Mean (SD) }\end{array}$ & $\begin{array}{l}\text { Group } \\
\text { difference } \\
\text { p-value }\end{array}$ \\
\hline Sex, male/female & $13 / 33$ & $10 / 22$ & \\
Age, years & $28.32(8.55)$ & $27.19(10.29)$ & 0.60 \\
Handedness, R/L & $46 / 0$ & $32 / 0$ & \\
HDRS & $29.17(6.23)$ & $1.125(1.18)$ & 0.00001 \\
LES-pos & $7.20(I 1.66)$ & $6.0313(9.79)$ & 0.65 \\
LES-neg & $77.98(69.43)$ & $3.6563(7.7 I)$ & 0.00001 \\
LES-total & $85.37(71.88)$ & $9.69(13.20)$ & 0.00001 \\
WCCL-avoidance & $0.63(0.19)$ & $0.29(0.18)$ & $0.0000 \mathrm{I}$ \\
WCCL-fantasy & $0.55(0.19)$ & $0.25(0.18)$ & $0.0000 \mathrm{I}$ \\
WCCL-remorse & $0.67(0.24)$ & $0.14(0.15)$ & $0.0000 \mathrm{I}$ \\
WCCL-seek help & $0.37(0.27)$ & $0.59(0.26)$ & 0.0007 \\
WCCL-rationalization & $0.48(0.19)$ & $0.30(0.14)$ & 0.00005 \\
WCCL-solve-pro & $0.47(0.25)$ & $0.82(0.16)$ & $0.0000 \mathrm{I}$ \\
\hline
\end{tabular}

Note: Data are presented as the mean (SD).

Abbreviations: MDD, major depressive disorder; HC, healthy controls; HDRS, 24-item Hamilton Rating Scale for depression; R, right; L, left. LES, the Life Events Scale; WCCL, the Ways of Coping Checklist; LES-pos, positive life events as measured by the LES; LES-neg, negative life events.

suicidal tendency; 3) serious medical or neurological illness; 4) substance dependence including tobacco, alcohol, or other psychoactive substances; or 5) metallic implants or other contraindications to MRI. All study procedures were conducted in accordance with the Declaration of Helsinki on Ethical Principles and approved by the local Institutional Review Boards of Hangzhou Normal University. All subjects provided written informed IRB-approved consent before participating in study procedures.

\section{MRI Data Acquisition}

All MRI data were acquired on a 3.0 T Discovery MR 750 scanner (General Electric, Waukesha, WI, USA) at the Center for Cognition and Brain Disorders at Hangzhou Normal University. During the MRI scan, a comfortable and tight cushion was placed to immobilize the head and reduce motion. The participants were instructed to relax and remain still with their eyes close, not to fall asleep, and not to think about anything in particular. Functional images were obtained in an interleaved order using a $\mathrm{T} 2 *$-weighted gradient-echo EPI pulse sequence. The acquisition parameters were as follows: $\mathrm{TR} / \mathrm{TE}=2000 / 22$, flip angle $=$ $77^{\circ}$, field of view $=240 \times 240 \mathrm{~mm}^{2}$, matrix $=96 \times 96$, $2.5 \mathrm{~mm}$ isotropic spatial resolution with 42 slices and 240 volumes. High-resolution T1-weighted anatomical images were also obtained in sagittal orientation for visualization and localization of the functional data with fast spoiled gradient echo sequence (3D FSPGR): TR/TE $=9 / 3.66$, flip angle $=13^{\circ}$, field of view $=240 \times 240 \mathrm{~mm}^{2}$, matrix $=$ $300 \times 300,0.8 \mathrm{~mm}$ isotropic voxels, 176 slices without interslice gap.

\section{MRI Preprocessing}

MRI data processing was performed using a combination of DPABI (http://www.rfmri.org/), SPM (http://www.fil.ion. ucl.ac.uk/spm/) and custom code written in MATLAB. After discarding the first 10 volumes for magnetization equilibration effects and the MRI environmental adaptation, the functional images were corrected for the time shifts among different acquisitions within each volume by sinc interpolating volume slices. Each volume was then realigned to the first volume to estimate motion parameters and correct for head motion. All participants had a maximum displacement of less than $2 \mathrm{~mm}$ in the $\mathrm{x}-, \mathrm{y}-$, or z-axes and an angular motion of less than 2 during the entire MRI scan. The corrected images were spatially normalized to the standard stereotactic space of the Montreal Neurological Institute (MNI) using the transformation derived from $\mathrm{T} 1$ segmentation and resampled to $3-\mathrm{mm}$ isotropic voxels. The images generated were smoothed with a 6-mm full-width half-maximum Gaussian kernel. Finally, the images underwent temporal bandpass filtering (between 0.01 and $0.1 \mathrm{~Hz}$ ).

\section{Entropy And Functional Connectivity Calculations}

Sample entropy measures the irregularity of a data series by computing the negative natural logarithm of an estimate of the conditional probability for that epochs of length that matches point-wise within a tolerance value that also matches at the next point. Brain entropy maps were generated on a voxel-by-voxel basis using the sample entropy approach ${ }^{28}$ of the Brain Entropy Mapping Toolbox (BENtbx, https://cfn. upenn.edu/ zewang/BENtbx.php). Based on previous optimal parameter values for R-fMRI data, ${ }^{23,29}$ the window length $(\mathrm{m})$ was set to 3 , and the cut-off tolerance threshold (r) was set to 0.6 multiplied by the standard deviation of the data. In the present study, we computed brain entropy on a voxel-by-voxel basis in the whole brain and averaged brain entropy across all voxels within a given brain mask or ROI for between-group comparisons. We also analysed the relationship between the voxel-wise brain entropy and HDRS scores in the MDD group. 
Functional connectivity was computed for each voxel according to a previous study. ${ }^{30}$ The representative time courses of each individual ROI within the thalamus were extracted by averaging the R-fMRI time series over all voxels in that region, and the RSFC strengths were calculated between each subregion and other voxels in the whole brain using Pearson correlation of their R-fMRI time series. ${ }^{31}$ The RSFC map was converted using Fisher's $\mathrm{r}$ to $\mathrm{z}$ transformation to improve the Gaussian distribution for group level analyses.

\section{Automated Functional Subdivision In The Thalamus}

To examine functional heterogeneity and homogeneity within the thalamus, we adopted the Louvain method ${ }^{32}$ for network module detection based on graph theory ${ }^{33}$ to automatically segment the thalamus into subregions. First, a thalamic mask was defined by the automated anatomical labelling (AAL) atlas bilaterally. ${ }^{34}$ The R-fMRI time series of all voxels within the thalamic mask were extracted, and Pearson's correlation coefficients were computed between all possible pairs of voxels to form a symmetric matrix. Second, the backbone of the group mean matrix was then constructed by applying a nonparametric method of locally adaptive network sparsification, ${ }^{35}$ and the module detection algorithm ${ }^{36}$ was applied to the backbone to parcellate the whole thalamus into composite modules for further functional connectivity analysis.

\section{Statistical analysis}

Between-group differences in the entropy and RSFC values were conducted on a voxel-by-voxel basis in the whole brain using the two-sample $t$-test. A multiple comparison of Gaussian random field theory (GRF) was performed with voxel $\mathrm{p}<0.005$, and a GRF cluster-corrected threshold of $p<0.05$. Given their ambiguous biological interpretations of negative RSFC, the between-group statistical comparisons were restricted to positive RSFC, and all negative ones were removed. Pearson's correlation analysis between the entropy map and HDRS scores in the MDD group was performed on a voxel-by-voxel basis in the whole brain, and the statistical threshold at cluster $\mathrm{p}$ $<0.05$ and correction threshold of multiple comparisons using GRF at voxel $p<0.005$ were set. In addition, ROI analysis and between-group comparison for entropy data were also conducted based on the whole thalamus and seven masks derived from the aforementioned thalamic subdivision of the $\mathrm{HC}$ group.

\section{Results}

\section{Between-Group Differences In Resting- State Brain Entropy}

Compared with healthy participants, MDD patients had significantly decreased brain entropy primarily in the bilateral thalami (peak MNI coordinates, left side at $\mathrm{x}=-18, \mathrm{y}=-15$, $\mathrm{z}=12, \mathrm{t}=-4.68$, cluster size $=110$ voxels; right side at $\mathrm{x}=18$, $\mathrm{y}=-15, \mathrm{z}=0, \mathrm{t}=-4.62$, cluster size $=76$ voxels) extending to the bilateral insula, bilateral putamen, left caudate and right inferior frontal gyrus $(p<0.05$, corrected; Figure 1). The t-maps from the aforementioned between-group comparison were converted into a mask for computing the mean entropy. MDD patients had significantly decreased mean entropy than the controls $(\mathrm{t}=-5.67, \mathrm{p}<0.00001)$.

\section{Correlations Between The Entropy Values And Clinical Features Of MDD}

As shown in Figure 2, there were significant negative correlations between the resting-state brain entropy and HDRS scores primarily in the left superior temporal gyrus (STG, correlation coefficient $r$ at peak voxel peak was -0.58 , MNI coordinates: $\mathrm{x}=-48, \mathrm{y}=15, \mathrm{z}=-9$, cluster size $=110$

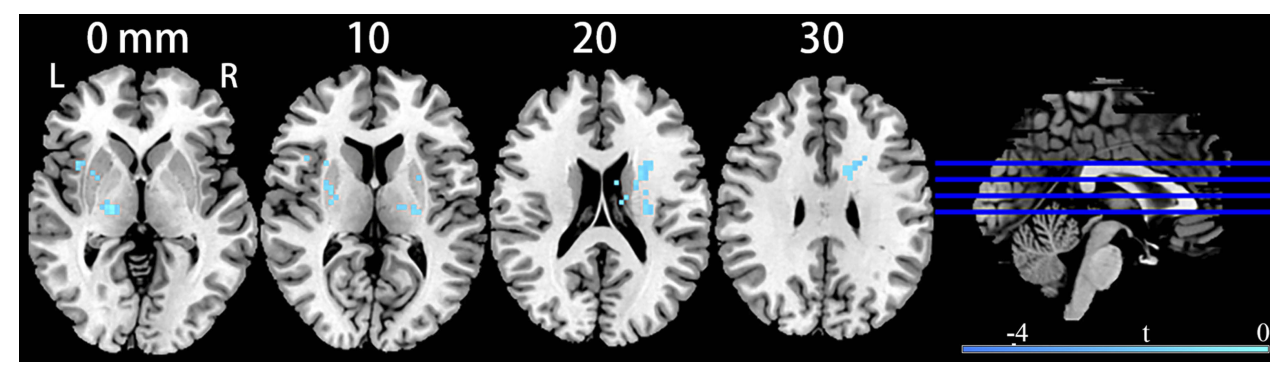

Figure I Clusters showing brain entropy differences in MDD patients compared with HC. Significance level was defined at voxel $\mathrm{p}<0.005$, cluster $\mathrm{p}<0.05$, GRF corrected. $L$ refers to the left side of the images in the coronal, and horizontal sections correspond to the left side of the brain. 


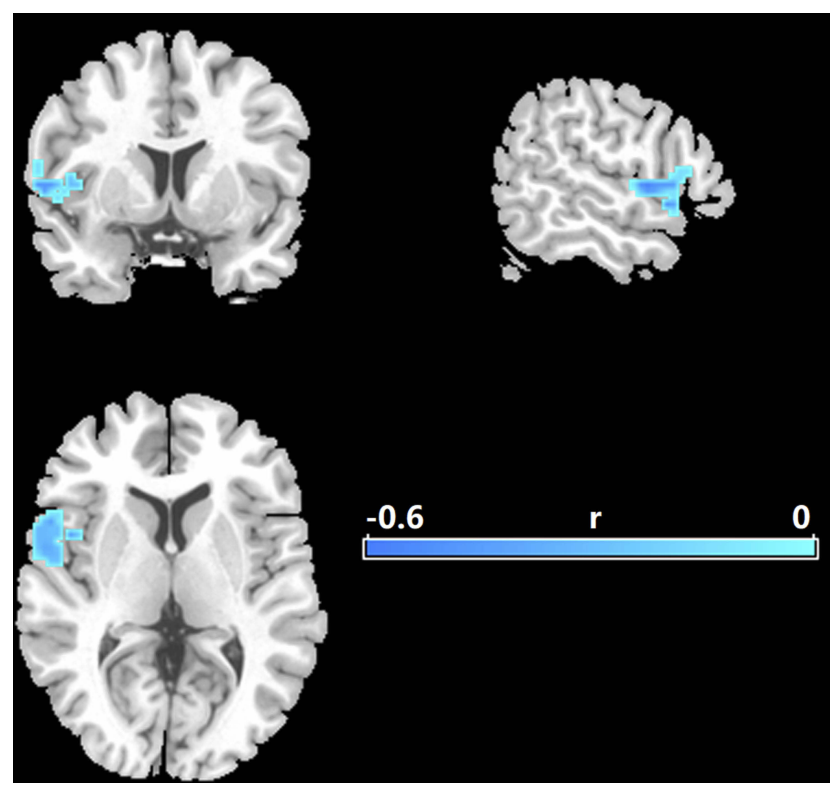

Figure 2 Correlations between entropy and the Hamilton rating scale for MDD. Significance level was defined at voxel $p<0.005$, cluster $p<0.05$, GRF corrected. L refers to the left side of the images in the coronal, and horizontal sections correspond to the left side of the brain.

voxels), which extended to the left Rolandic operculum, left inferior frontal gyrus, and left insula ( $p<0.05$, corrected).

\section{Functional Parcellation Within The Thalamus}

To examine the subregional specificity within the whole thalamus, the module detection method ${ }^{36}$ was used based on the R-fMRI voxel time series within the bilateral thalami. The modularity structure was observed in both groups $(\mathrm{Q}=0.57$ for the $\mathrm{HC}$ group and $\mathrm{Q}=0.59$ for the
MDD group). Seven subregions (ROI) were detected in the HC group (Figure 3A: ROI 1, red; ROI 2, green; ROI 3, blue; ROI 4, orange; ROI 5, cyan; ROI 6, yellow; ROI 7, magenta). In the MDD group, seven subregions were also detected (Figure 3B: ROI 1, red; ROI 2, green; ROI 3, blue; ROI 4, orange; ROI 5, cyan; ROI 6, yellow; ROI 7, magenta). The subdivisions of the thalamus identified in the MDD and HC groups were very similar, and further entropy comparison and RSFC analysis were processed based on the ROI patterns of the thalamus in the $\mathrm{HC}$ group. As shown in Figure 4, MDD patients had significantly decreased mean entropy compared with HC (two-sample $t$-test, ROI $1, \mathrm{t}=-1.53, \mathrm{p}=0.13 ;$ ROI $2, \mathrm{t}=-2.27, \mathrm{p}=0.03$; ROI 3, $\mathrm{t}=-2.71, \mathrm{p}=0.008$; ROI $4, \mathrm{t}=-1.48, \mathrm{p}=0.14$; ROI $5, \mathrm{t}=-2.37, \mathrm{p}=0.02$; ROI $6, \mathrm{t}=-3.63, \mathrm{p}=0.00$; ROI 7 , $\mathrm{t}=-2.19, \mathrm{p}=0.03$; the whole thalamus, $\mathrm{t}=-2.63, \mathrm{p}=0.01$ ).

\section{Altered Functional Connectivity Of Thalamic Subregions In Major Depressive Disorder}

The RSFC comparison results are shown in Figure 5 and Table 2. Compared with HC, MDD patients indicated significantly increased RSFC between ROI 1 and the left medial part of the superior frontal gyrus (mSFG), which extended to the left inferior and middle prefrontal cortex, left anterior and middle cingulate cortex, and left precentral gyrus $(\mathrm{p}<0.05$, corrected; Figure 5$)$. The ROI 2 in MDD patients exhibited significantly increased RSFC with the bilateral $\mathrm{mSFG}$, which extended to the anterior cingulate cortex, caudate, and putamen. We also observed that

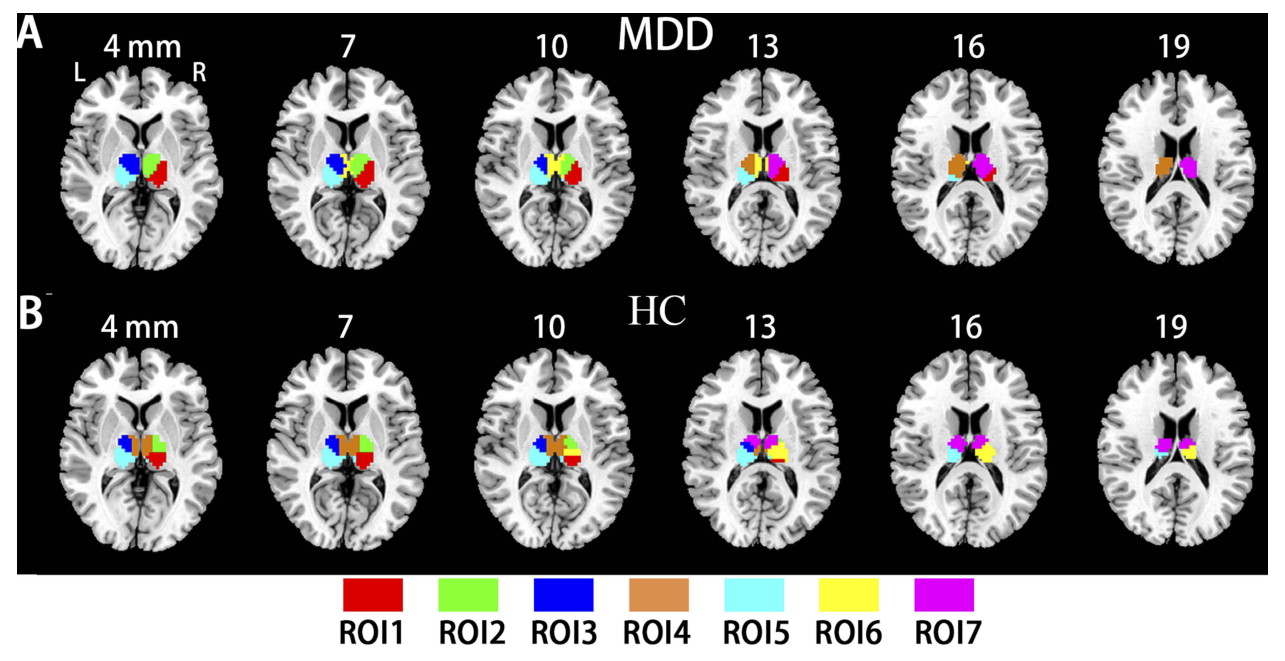

Figure 3 The thalamic subregions. (A) Automated detection of seven thalamic subregions in the HC group. (B) Automated detection of seven thalamic subregions in the MDD group. 


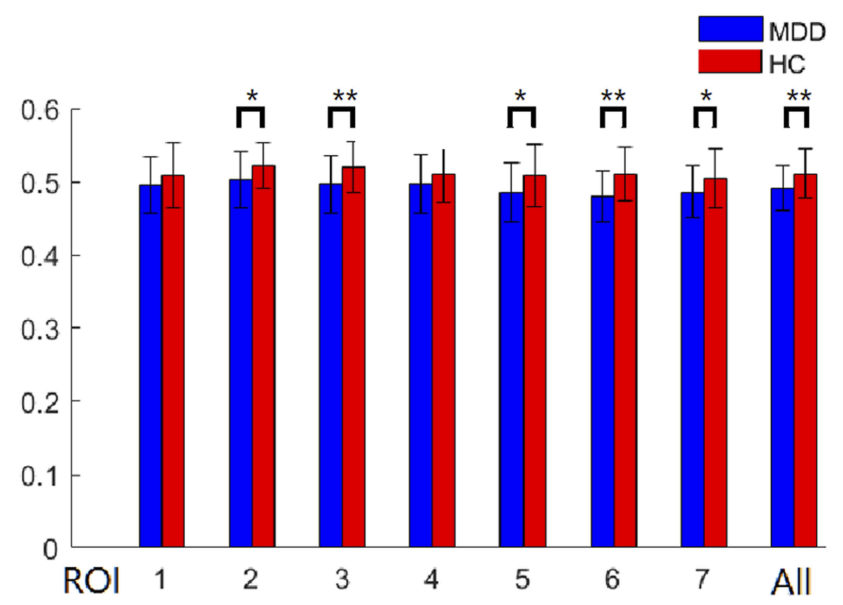

Figure 4 Between-group differences of subregional mean entropy. All, bilateral whole thalamus. $*_{\mathrm{p}}<0.05 ; * * \mathrm{P}<0.01$.

Abbreviations: $\mathrm{ROI}$, region of interest; $\mathrm{BE}$, brain entropy.

ROI 2 exhibited significantly increased RSFC with the right insula, including the right caudate, right putamen, and right Rolandic operculum. ROI 3 had significantly increased RSFC with the bilateral $\mathrm{mSFG}$ and left inferior orbital frontal gyrus, including the left insula. ROI 4 showed significantly increased RSFC with the bilateral mSFG. ROI 5 showed significantly increased RSFC with five clusters, including the bilateral $\mathrm{mSFG}$, bilateral STG, bilateral Rolandic operculum and bilateral heschl. ROI 6 showed significantly increased RSFC with the bilateral mSFG and bilateral putamen. ROI 7 showed significantly increased RSFC with the bilateral $\mathrm{mSFG}$, left inferior orbital frontal gyrus, left Rolandic operculum and bilateral insula.

\section{Discussion}

The present study provides the first evidence of restingstate brain entropy changes in patients with MDD. The results showed that decreased entropy in MDD was mainly located in anterior subregions of the thalamus. Automated functional subdivision in the whole thalamus was implemented based on R-fMRI data using a Louvain method for module detection. We subdivided the whole thalamus into seven subregions and identified distinct RSFC patterns. MDD patients showed that the brain entropy in the STG was correlated with the HDRS scores.

The thalamus is a central brain region that plays a crucial role in relaying and integrating information across neural systems underlying awareness and sensory, motor, and cognitive functions. ${ }^{37}$ Reduced entropy in the thalamus implied that the dynamic activity of this region in
MDD patients was organized towards a regular or single state. It may lead to insufficient types of brain activity patterns to meet diverse functional requirements and even a loss of flexibility in relaying various incoming and outgoing information to appropriate regions, which also implies that the thalamus is an important region for the neuropathology in MDD. This finding about the thalamus provides new evidence from the perspective of temporal brain activity dynamics that complements previous studies that have reported thalamic dysfunction in MDD. ${ }^{14}$

In recent years, there has been growing particular interest in examining thalamic dysfunction with the delineation of thalamic parcellation and various RSFC patterns. ${ }^{38,39,40}$ The thalamus is a communication conduit between subcortical brain areas and the cerebral cortex, such as in the thalamo-cortical system. Previous research has shown that impaired thalamo-cortical processing is involved in MDD. ${ }^{14}$ Our results of an abnormal enhancement of RSFC between the thalamus and $\mathrm{mSFG}$ suggested disturbances of MDD-related thalamo-cortical functional coupling. The results also demonstrated that the RSFC alterations with the $\mathrm{mSFG}$ in MDD were widely distributed across almost all thalamic subregions, suggesting a general involvement of the thalamic independent of the location and function of its subregions.

MDD patients exhibited distinct RSFC patterns of thalamic subregions. ROI 5 showed significantly increased RSFC, with peak differences in the STG and Heschl's sulcus (also called the transverse temporal gyri), suggesting that abnormal RSFC between ROI 5 and the temporal lobe might play an important role in the pathophysiology of MDD. This postulation was consistent with previous MRI studies ${ }^{41}$ and our clinical association analysis results between the brain entropy and HDRS scores. The STG plays a crucial role in the processing of emotional experiences and social cognition and has been repeatedly implicated in MDD. ${ }^{42}$ For example, adolescents with a history of suicide attempt and treatment-resistant depression had decreased STG volume compared with HC. ROIs 5 and 7 showed significantly increased RSFC with Rolandic operculum. The Rolandic operculum belongs to the auditory network and has been detected as a feature with discriminative power when classifying MDD patients from $\mathrm{HC}{ }^{43}$ ROIs 2 and 7 showed significantly increased RSFC with insula. Anatomically, the insula (often called the insular cortex) is part of the cerebral cortex folded deep within the lateral sulcus, which receives input from thalamic nuclei. The bilateral insular cortex is related to self-awareness and 


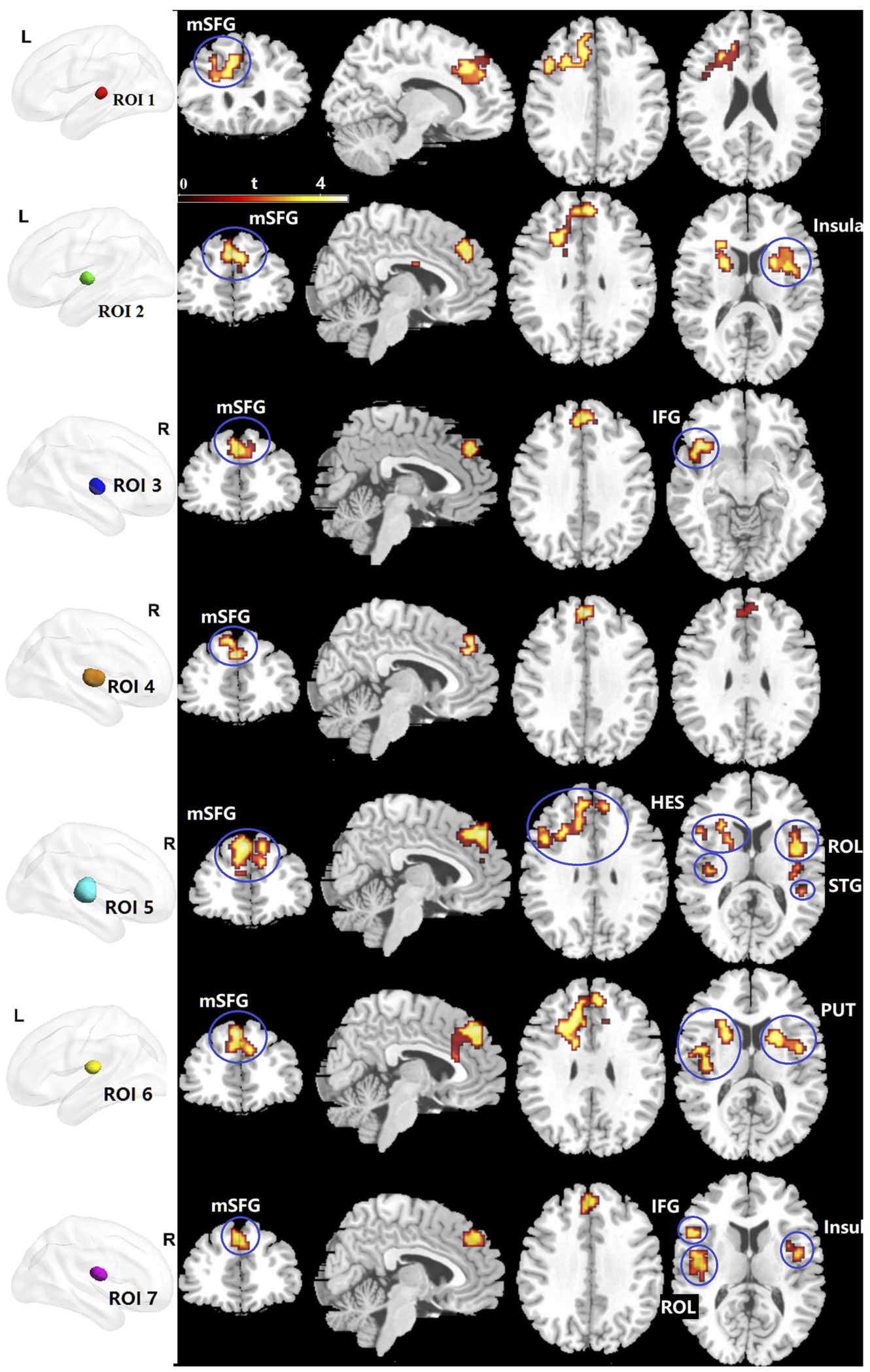

Figure 5 Clusters showing between-group RSFC differences of the thalamic subregions. Significance level was defined at voxel $p<0.005$, cluster $p<0.05$, GRF corrected. R refers to the left side of the image in the coronal and horizontal sections corresponding to the left side of the brain.

Abbreviations: ROI, regions of interest; mSFG, the medial part of the superior frontal gyrus; IFG, inferior orbital frontal gyrus; HES, Heschl; ROL, Rolandic operculum; STG, superior temporal gyrus; PUT, putamen; L, left side. 
Table 2 Altered RSFC Of Thalamic Subregions In MDD Patients

\begin{tabular}{|c|c|c|c|c|}
\hline $\begin{array}{l}\text { Anatomical } \\
\text { region }\end{array}$ & Colour & $\begin{array}{l}\text { Cluster } \\
\text { Size } \\
\text { (voxels) }\end{array}$ & $\begin{array}{l}\text { Peak } \\
\text { t-value }\end{array}$ & $\begin{array}{l}\text { Peak MNI (XY } \\
\text { Z) coordinates } \\
(\mathrm{mm})\end{array}$ \\
\hline ROI I & \multicolumn{4}{|l|}{ Red } \\
\hline L mSFG & & 316 & 4.5 & $-9,27,36$ \\
\hline ROI 2 & \multicolumn{4}{|l|}{ Green } \\
\hline B mSFG & & 287 & 4.5 & $0,42,33$ \\
\hline$R$ insula & & 153 & 4.18 & $45,-6,3$ \\
\hline ROI 3 & \multicolumn{4}{|l|}{ Blue } \\
\hline B mSFG & & 80 & 4.32 & $0,42,33$ \\
\hline L IFG & & 72 & 4.9 & $-42,18,-12$ \\
\hline ROI 4 & \multicolumn{4}{|l|}{ Orange } \\
\hline B mSFG & & 69 & 4.04 & $0,45,33$ \\
\hline ROI 5 & \multicolumn{4}{|l|}{ Cyan } \\
\hline B mSFG & & 729 & 5.22 & $-6,42,42$ \\
\hline R STG & & 123 & 4.84 & $60,-12,0$ \\
\hline L HES & & 113 & 4.34 & $-39,-18,6$ \\
\hline R ROL & & 122 & 4.5 & $42,-3,12$ \\
\hline R ROL & & 83 & 4.1 & $45,-24,21$ \\
\hline ROI 6 & \multicolumn{4}{|l|}{ Yellow } \\
\hline B mSFG & & 731 & 4.51 & $-3,42,42$ \\
\hline R PUT & & 98 & 4.29 & $24,9,12$ \\
\hline ROI 7 & \multicolumn{4}{|l|}{ Magenta } \\
\hline B mSFG & & 73 & 4.40 & $-3,45,39$ \\
\hline$R$ insula & & 54 & 4.05 & $45,-6,3$ \\
\hline L ROL & & 122 & 4.03 & $-48,-6,9$ \\
\hline L IFG & & 221 & 5.39 & $-39,15,-9$ \\
\hline
\end{tabular}

Notes: Significance level was defined at voxel $p<0.005$, cluster $p<0.05$, GRF corrected.

Abbreviations: RSFC, resting-state functional connectivity; MDD, major depressive disorder; R, right side; L, left side; B; bilateral side; MNI, Montreal Neurological Institute coordinates; mSFG, the medial part of the superior frontal gyrus; IFG, inferior orbital frontal gyrus; HES, Heschl; ROL, Rolandic operculum; STG, superior temporal gyrus; PUT, putamen; ROI, region of interest.

to the processing of emotional information, which are frequently affected in MDD. ${ }^{44}$ Previous MRI studies have reported that the insula could be a key region associated with depression. ${ }^{45,46}$ One possibility is that increased RSFC between the anterior thalamus and bilateral insula underlies emotion processing under MDD. ROIs 7 and 3 showed significantly increased RSFC with the inferior orbital frontal gyrus. A previous study reported that cerebral glucose metabolism in the inferior orbital frontal gyrus of MDD patients was correlated with depressive symptoms. ${ }^{47}$
Thalamus-related brain networks contain two components, i.e., regional neural properties and how the regional activities interact with others in the brain. ${ }^{48}$ Decreased entropy in the thalamus showed abnormal local regional spontaneous neural activities in MDD, while increased thalamic RSFC with the mSFG and other cortical regions indicated disturbances of MDD-related thalamo-cortical functional coupling. MDD patients exhibited this reverse abnormal tendency between the two results, indicating a single state of regional activities in the thalamus was accompanied by an abnormal enhancement of RSFC with the cerebral cortex.

The present study has two limitations. First, the present study is preliminary. Further studies with a larger sample size are expected to confirm the reproducibility of these findings. Second, the coordination of cortical and thalamic activity plays a vital role in regulating the state of sleep and wakefulness. The present exploratory study showed brain entropy and thalamic functional connectivity during the eyes-closed resting-state scans, and the participants were instructed to remain still and not to fall asleep in scan. Further R-fMRI studies can benefit by optimizing the experimental design (e.g., including a sleep monitor). These data are preliminary and require replication with larger numbers of subjects.

\section{Conclusion}

Overall, our study demonstrated a resting-state brain entropy reduction in anterior subregions of the thalamus in MDD patients. Increased RSFC between the thalamus and $\mathrm{mSFG}$ were also found in MDD patients. The findings suggested entropy as a potential marker that represents a novel perspective on the temporal dynamics of functional brain activity and enhanced the understanding of the various pathophysiologic processes in MDD.

\section{Acknowledgments}

We would like to acknowledge the support of Prof. YuFeng Zang and $\mathrm{Xu}-\mathrm{Chu}$ Weng. This study was supported by the National Natural Science Foundation of China (81271503), the Natural Science Foundation of Zhejiang Province (LY17H180007), the Hangzhou Science and Technology Commission Foundation (20170533B06), the Scientific Research Start-up Foundation (PF15002004049) and the Cultivation Project of the Province-level Preponderant Characteristic Discipline in the College of Education (18JYXK036) of Hangzhou Normal University. 


\section{Disclosure}

The authors report no conflicts of interest in relation to this work.

\section{References}

1. Richards CS, O'Hara MW. The Oxford Handbook of Depression and Comorbidity. Oxford University Press; 2014:254.

2. Kessler RC, Berglund P, Demler O, Jin R, Merikangas KR, Walters EE. Lifetime prevalence and age-of-onset distributions of DSM-IV disorders in the National Comorbidity Survey Replication. Arch Gen Psychiatry. 2005;62:593-602. doi:10.1001/archpsyc.62.6.593

3. Belzung C, Willner P, Philippot P. Depression: from psychopathology to pathophysiology. Curr Opin Neurobiol. 2015;30:24-30. doi:10.1016/j.conb.2014.08.013

4. Graham J, Salimi-Khorshidi G, Hagan C, et al. Meta-analytic evidence for neuroimaging models of depression: state or trait? J Affect Disord. 2013;151:423-431. doi:10.1016/j.jad.2013.07.002

5. Gong Q, He Y. Depression, neuroimaging and connectomics: a selective overview. Biol Psychiatry. 2015;77:223-235. doi:10.1016/j. biopsych.2014.08.009

6. Busatto GF. Structural and functional neuroimaging studies in major depressive disorder with psychotic features: a critical review. Schizophr Bull. 2013;39:776-786. doi:10.1093/schbul/sbt054

7. Hahn T, Marquand AF, Ehlis AC, et al. Integrating neurobiological markers of depression. Arch Gen Psychiatry. 2011;68:361-368. doi:10.1001/archgenpsychiatry.2010.178

8. Lopez-Larson M, King JB, McGlade E, et al. Enlarged thalamic volumes and increased fractional anisotropy in the thalamic radiations in veterans with suicide behaviors. Front Psychiatry. 2013;4:83. doi:10.3389/fpsyt.2013.00083

9. Yuan R, Di X, Taylor PA, Gohel S, Tsai YH, Biswal BB. Functional topography of the thalamocortical system in human. Brain Struct Funct. 2016;221:1971-1984. doi:10.1007/s00429-015-1018-7

10. Bora E, Harrison BJ, Davey CG, Yucel M, Pantelis C. Meta-analysis of volumetric abnormalities in cortico-striatal-pallidal-thalamic circuits in major depressive disorder. Psychol Med. 2012;42:671-681. doi:10.1017/S0033291711001668

11. Seminowicz DA, Mayberg HS, McIntosh AR, et al. Limbic-frontal circuitry in major depression: a path modeling metanalysis Neuroimage. 2004;22:409-418. doi:10.1016/j.neuroimage.2004.01.015

12. Kumar VJ, van Oort E, Scheffler K, Beckmann CF, Grodd W. Functional anatomy of the human thalamus at rest. Neuroimage. 2017;147:678-691. doi:10.1016/j.neuroimage.2016.12.071

13. Clemente-Perez A, Makinson SR, Higashikubo B, et al. Distinct thalamic reticular cell types differentially modulate normal and pathological cortical rhythms. Cell Rep. 2017;19:2130-2142. doi:10.1016/j.celrep.2017.05.044

14. Brown EC, Clark DL, Hassel S, MacQueen G, Ramasubbu R. Thalamocortical connectivity in major depressive disorder. $J$ Affect Disord. 2017;217:125-131. doi:10.1016/j.jad.2017.04.004

15. Wolff M, Alcaraz F, Marchand AR, Coutureau E. Functional heterogeneity of the limbic thalamus: from hippocampal to cortical functions. Neurosci Biobehav Rev. 2015;54:120-130. doi:10.1016/j. neubiorev.2014.11.011

16. Kong QM, Qiao H, Liu CZ, et al. Aberrant intrinsic functional connectivity in thalamo-cortical networks in major depressive disorder. CNS Neurosci Ther. 2018;24:1063-1072. doi:10.1111/cns.12831

17. Saxe GN, Calderone D, Morales LJ. Brain entropy and human intelligence: a resting-state fMRI study. PLoS One. 2018;13: e0191582. doi:10.1371/journal.pone.0191582

18. Arturo T, Marzieh Z, Benasich AA. New perspectives on spontaneous brain activity: dynamic networks and energy matter. Front Hum Neurosci. 2016;10:00247.
19. Pezard L, Martinerie J, Müller-Gerking J, Varela FJ, Renault B. Entropy quantification of human brain spatio-temporal dynamics. Physica D. 1996;96:344-354. doi:10.1016/0167-2789(96)00032-2

20. Wang Z, Li Y, Childress AR, Detre JA. Brain entropy mapping using fMRI. PLoS One. 2014;9:e89948. doi:10.1371/journal. pone. 0089948

21. Xue SW, Yu Q, Guo Y, Song D, Wang Z. Resting-state brain entropy in schizophrenia. Compr Psychiatry. 2019;89:16-21. doi:10.1016/j. comppsych.2018.11.015

22. Xue SW, Guo Y. Increased resting-state brain entropy in Alzheimer's disease. Neuroreport. 2018;29:286-290. doi:10.1097/WNR.00000000 00000942

23. Zhou F, Zhuang Y, Gong H, Zhan J, Grossman M, Wang Z. Resting state brain entropy alterations in relapsing remitting multiple sclerosis. PLoS One. 2016;11:e146080. doi:10.1371/journal.pone.0146080

24. Sokunbi MO, Fung W, Sawlani V, Choppin S, Linden DE, Thome J. Resting state fMRI entropy probes complexity of brain activity in adults with ADHD. Psychiatry Res. 2013;214:341-348. doi:10.1016/ j.pscychresns.2013.10.001

25. Lener MS, Iosifescu DV. In pursuit of neuroimaging biomarkers to guide treatment selection in major depressive disorder: a review of the literature. Ann N Y Acad Sci. 2015;1344:50-65. doi:10.1111/ nyas.2015.1344.issue-1

26. Drevets WC, Bogers W, Raichle ME. Functional anatomical correlates of antidepressant drug treatment assessed using PET measures of regional glucose metabolism. Eur Neuropsychopharmacol. 2002;12:527-544. doi:10.1016/S0924-977X(02)00102-5

27. Oldfield RC. The assessment and analysis of handedness: the Edinburgh inventory. Neuropsychologia. 1971;9:97-113. doi:10.1016/0028-3932 (71) $90067-4$

28. Richman JS, Moorman JR. Physiological time-series analysis using approximate entropy and sample entropy. Am J Physiol Heart Circ Physiol. 2000;278:H2039-H2049. doi:10.1152/ajpheart.2000.278.6. H2039

29. Li Z, Fang Z, Hager N, Rao H, Wang Z. Hyper-resting brain entropy within chronic smokers and its moderation by sex. Sci Rep. 2016;6:29435. doi:10.1038/srep29435

30. Margulies DS, Kelly AM, Uddin LQ, Biswal BB, Castellanos FX, Milham MP. Mapping the functional connectivity of anterior cingulate cortex. Neuroimage. 2007;37:579-588. doi:10.1016/j.neuroimage.2007.05.019

31. Biswal BB, Van Kylen J, Hyde JS. Simultaneous assessment of flow and BOLD signals in resting-state functional connectivity maps. NMR Biomed. 1997;10:165-170. doi:10.1002/(ISSN)1099-1492

32. Blondel VD, Guillaume JL, Lambiotte R, Lefebvre E. Fast unfolding of communities in large networks. J Stat Mech-Theory E. 2008;10: P10008. doi:10.1088/1742-5468/2008/10/P10008

33. Xia M, Wang Z, Dai Z, et al. Differentially disrupted functional connectivity in posteromedial cortical subregions in Alzheimer's disease. J Alzheimers Dis. 2014;39:527-543. doi:10.3233/JAD-131583

34. Tzourio-Mazoyer N, Landeau B, Papathanassiou D, et al. Automated anatomical labeling of activations in SPM using a macroscopic anatomical parcellation of the MNI MRI single-subject brain. Neuroimage. 2002;15:273-289. doi:10.1006/nimg.2001.0978

35. Foti NJ, Hughes JM, Rockmore DN. Nonparametric sparsification of complex multiscale networks. PLoS One. 2011;6:e16431. doi:10.1371/journal.pone.0016431

36. Newman ME. Modularity and community structure in networks. Proc Natl Acad Sci USA. 2006;103:8577-8582. doi:10.1073/ pnas.0601602103

37. Hwang K, Bertolero MA, Liu WB, D’Esposito M. The human thalamus is an integrative hub for functional brain networks. $J$ Neurosci. 2017;37:5594-5607. doi:10.1523/JNEUROSCI.0067-17.2017

38. Glaister J, Carass A, NessAiver T, et al. Thalamus segmentation using multi-modal feature classification: validation and pilot study of an age-matched cohort. Neuroimage. 2017;158:430-440. doi:10.1016/j.neuroimage.2017.06.047 
39. Hale JR, Mayhew SD, Mullinger KJ, et al. Comparison of functional thalamic segmentation from seed-based analysis and ICA. Neuroimage. 2015;114:448-465. doi:10.1016/j.neuroimage.2015.04.027

40. Lambert C, Simon H, Colman J, Barrick TR. Defining thalamic nuclei and topographic connectivity gradients in vivo. Neuroimage. 2017;158:466-479. doi:10.1016/j.neuroimage.2016.08.028

41. Takahashi T, Yucel M, Lorenzetti V, et al. An MRI study of the superior temporal subregions in patients with current and past major depression. Prog Neuropsychopharmacol Biol Psychiatry. 2010;34:98-103. doi:10.1016/j.pnpbp.2009.10.005

42. McLellan Q, Wilkes TC, Swansburg R, Jaworska N, Langevin LM, MacMaster FP. History of suicide attempt and right superior temporal gyrus volume in youth with treatment-resistant major depressive disorder. J Affect Disord. 2018;239:291-294. doi:10.1016/j.jad.2018.07.030

43. Qin J, Wei M, Liu H, et al. Altered anatomical patterns of depression in relation to antidepressant treatment: evidence from a pattern recognition analysis on the topological organization of brain networks. J Affect Disord. 2015;180:129-137. doi:10.1016/j.jad.2015.03.059
44. Gu X, Hof PR, Friston KJ, Fan J. Anterior insular cortex and emotional awareness. J Comp Neurol. 2013;521:3371-3388. doi:10.1002/ cne. 23368

45. Yin Z, Chang M, Wei S, et al. Decreased functional connectivity in insular subregions in depressive episodes of bipolar disorder and major depressive disorder. Front Neurosci. 2018;12:842. doi:10.3389/fnins.2018.00842

46. Sliz D, Hayley S. Major depressive disorder and alterations in insular cortical activity: a review of current functional magnetic imaging research. Front Hum Neurosci. 2012;6:323. doi:10.3389/fnhum.2012. 00323

47. Wu JC, Gillin JC, Buchsbaum MS, et al. Sleep deprivation PET correlations of Hamilton symptom improvement ratings with changes in relative glucose metabolism in patients with depression. $J$ Affect Disord. 2008;107:181-186. doi:10.1016/j.jad.2007.07.030

48. Lee TW, Xue SW. Linking graph features of anatomical architecture to regional brain activity: a multi-modal MRI study. Neurosci Lett. 2017;651:123-127. doi:10.1016/j.neulet.2017.05.005

\section{Publish your work in this journal}

Neuropsychiatric Disease and Treatment is an international, peerreviewed journal of clinical therapeutics and pharmacology focusing on concise rapid reporting of clinical or pre-clinical studies on a range of neuropsychiatric and neurological disorders. This journal is indexed on PubMed Central, the 'PsycINFO' database and CAS, and is the official journal of The International Neuropsychiatric Association (INA). The manuscript management system is completely online and includes a very quick and fair peer-review system, which is all easy to use. Visit http://www.dovepress.com/testimonials.php to read real quotes from published authors. 